\title{
Exploration on the Application of Internet Online Courses in Modern Teaching of Guzheng
}

\author{
Yi Shen \\ Fujian Preschool Education College, Fujian, 350000, China
}

\begin{abstract}
As a traditional Chinese musical instrument, Guzheng wins favor from people since ancient times. In performance of Guzheng, its touching timbre and extensive everlasting rhyme create a strong sense of presence. Nowadays, since internet technologies have become the infrastructure in modern society, the paper studies how to achieve in-depth fusion of teaching of Guzheng (a representative of traditional culture) and the emerging internet technologies. In this paper, the advantages of introducing internet online courses into modern teaching of Guzheng are summarized, followed by a tentative analysis of application of internet online courses in modern teaching of Guzheng.
\end{abstract}

Keywords: Internet; Online Courses; Teaching of Guzheng; Innovation; Application.

\section{Internet Online Teaching Promotes the Learning of Guzheng}

According to the $48^{\text {th }}$ China Statistical Report on Internet Development issued by China Internet Network Information Center (CNNIC) in Beijing in August 2021, China's internet users increased by 21.75 million compared with December 2020 and reached 1.011 billion with an Internet penetration rate of $71.6 \%$, indicating that China has become the world's largest vivifying digital society. Since internet information technologies have become the infrastructure in modern society, the information dissemination in internet environment is characterized by timeliness, pervasiveness and diversity, etc. Internet online courses refers to in-depth fusion of traditional teaching mode into the internet environment, thus empowering the teaching concepts with internet technologies. Under the mode of internet online courses, the offline teaching is shifted to online teaching, forming a new teaching environment and content and bringing vitality to courses. Therefore, it's been a research hotspot in the new era concerning how to achieve in-depth fusion of teach of Guzheng (a representative of traditional culture) and the emerging internet technologies.

\section{The Advantages of Introducing Internet Online Courses into Modern Teaching of Guzheng}

\subsection{It Helps Improve Students' Efficiency in Learning of Guzheng}

In learning of Guzheng, students' knowledge about Guzheng is generally limited to superficial performance skills without much comprehension of the in-depth hidden meaning. As the capacity of Guzheng learners varies greatly, for learners who've learned playing Guzheng, the understanding of basic knowledge and history of Guzheng will help them improve performance skills. For those who lack the basics of Guzheng, they should not only learn to play Guzheng but also learn the theoretical knowledge, to gain an in-depth understanding of fingering of Guzheng.

The internet courses of Guzheng change the characteristics of its traditional teaching mode. Internet online courses are not limited by time and space: teachers upload the recorded teaching videos to the internet platform and students may learn Guzheng at any time and on any occasions. The teaching mode of internet online courses will narrow the learning gap among learners. Furthermore, students may carry out learning activities such as learning, review and performance at different time, thus greatly developing their interest in learning of Guzheng and improving the learning efficiency. 


\subsection{It Helps Improve Teachers' Teaching Method}

For children's Guzheng classes, the main teaching method is personal demonstration of teachers and students will imitate teachers' gesture and demonstration. After the end of class, teachers will assess children's learning achievement or point out what to be improved. However, such traditional teaching mode is relatively rigid: teachers are at a dominant position, while students are passively engaged in following teachers in learning with their imagination greatly restricted. Therefore, the students' learning initiatives cannot be well-cultivated. The teaching mode of internet online courses allows students to carry out targeted learning based on their own demand or learning situations. Meanwhile, internet enables more close ties and more frequent exchanges between students and teachers. Furthermore, teachers may also check students learning by holding concerts or performance Guzheng and give them the experience in performance of Guzheng. Thanks to internet, students will not only learn the knowledge of Guzheng available in the campus, but also the knowledge imparted by teachers outside the campus. To some extent, this achieves resources sharing and makes up for the inadequate development of teaching of Guzheng due to limited teaching resources.

\subsection{It Helps Improve Students' Artistic Emotions}

The teaching of Guzheng not only fuses the traditional teaching experience but also cultivate students' artistic emotions and pursuit. For instance, Fishing Boat in the Sunset is a typical Guzheng song in Henan which contains strong emotions. Therefore, in teaching this song, teachers should not only impart the performance skills of this song, but also covey the emotions of the song to students. Fishing Boat in the Sunset depicts a scene of life where fishmen are carefree in the background of sunset and water. The performance of Guzheng requires strong emotions and only in-depth understanding of the emotions or stories behind songs will a player give a consummate performance and add the soul to the songs. The internet online teaching mode enables the presentation of emotions behind songs to audience in form of scenes. With the pictures, students will feel immersive in the stories and scenes and gain a true and intimate feeling of the songs and their emotions. While changing the traditional boring teaching mode, internet online teaching brings vitality to theoretical teaching and improves students' learning interest in Guzheng and their imagination about songs as well, thus giving bettering play to their individual performance capacity.

\section{The Application of Internet Online Courses in Modern Teaching of Guzheng}

The application of internet technologies brings about convenience and innovation to modern teaching of Guzheng. According to author's practice in teaching of Guzheng, the application of internet online courses in modern teaching of Guzheng may divide the system into multiple teaching programs such as basic knowledge, basic skills, advanced skills, video and audio production appreciation and the performance given by renowned performers from the hierarchical structure of teaching content and Guzheng and from the practical experience of teaching. The teaching tasks and learning content vary with the teaching objects. Even for one student, the knowledge about Guzheng to be learned also differs at different learning stages. For students with certain basic knowledge about Guzheng, more difficult teaching may be conducted by selecting appropriate teaching content. For instance, in remote teaching or exchange programs, teachers and students may share their own insight with others. All students participating in online learning of Guzheng may watch the performance of Guzheng by renowned performers, imitate their performance skills and learn the performance after $\log$ in.

The design concept of "internet + teaching of Guzheng" is shown in Figure 1:

Guzheng is a traditional Chinese musical instrument. During performance, many details should be paid. For instance, the posture, gesture and wearing of artificial fingernails etc., during performance. Since Guzheng boasts a long history, it's worthwhile that all learners engage in-depth learning of its origin, inheritance, development and cultural connotation, etc. In order to help students better understand the characteristics of performance of Guzheng among different schools, internet online 
teaching of Guzheng provides songs for reference by each beginner, so that they may have a better start.

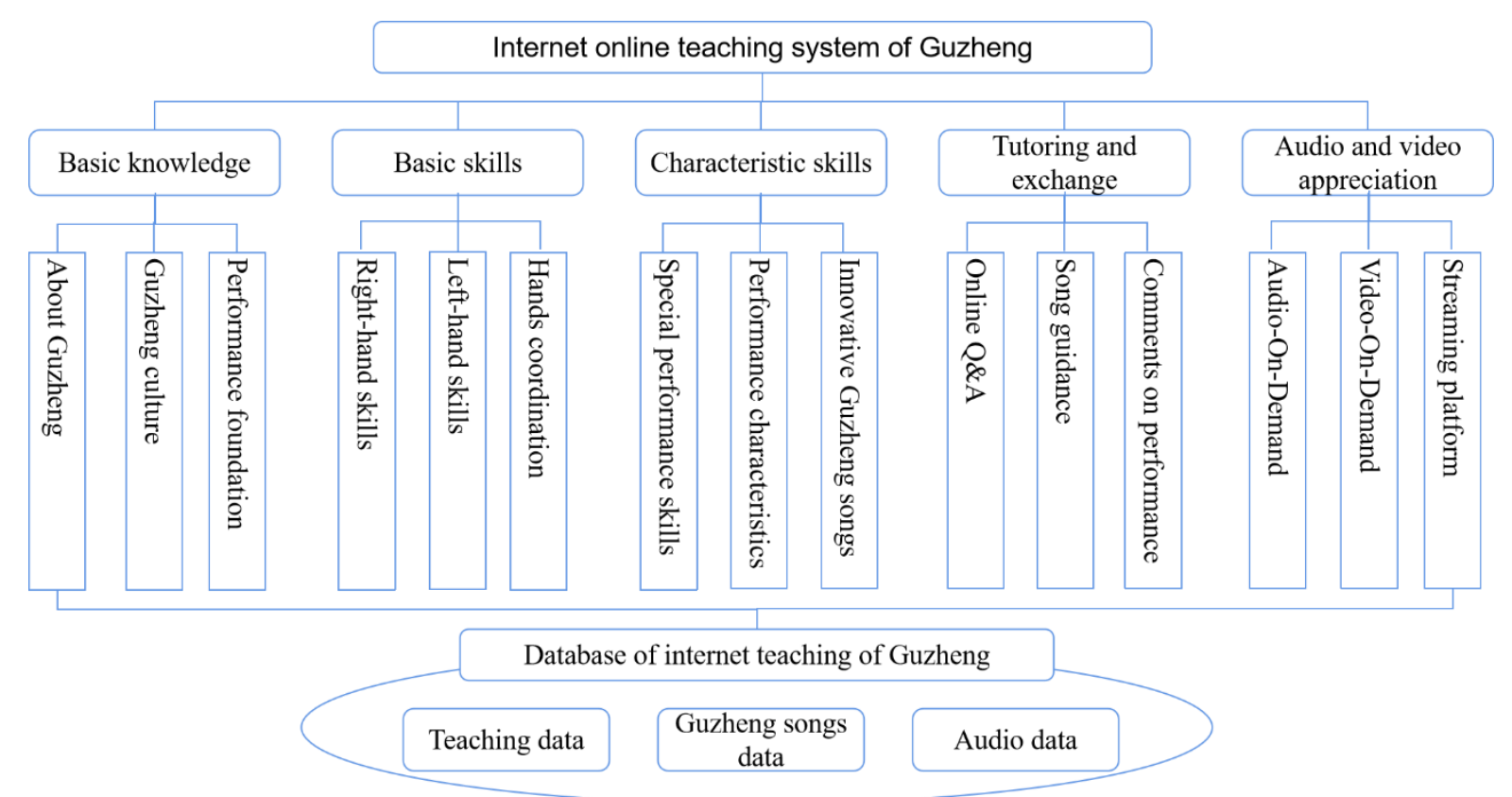

Figure 1. Design concept of "internet + teaching of Guzheng"

The primary performance skills of Guzheng are divided into three major categories: the right-hand skills, the left-hand skills and the hands coordination skills. During teaching of Guzheng, teachers will alternate the teaching of foregoing skills. At the same time, in order to arise students' learning interest, teachers will re-compose current popular songs into Guzheng songs, to help students acquaint themselves with the basic fingering skills.

After acquisition of basic skills, teachers must classify the songs by their performance skills and characteristics, etc. The teaching of advanced skills includes much content, among which Li tone, finger pointing, strumming, sweeping, performance styles and songs composition are the main content at this learning stage. The Guzheng songs of different schools may vary in terms of both content and style. In order to achieve better effect in teaching of Guzheng, teachers will fuse traditional teaching elements with innovative ones and combine regional cultural characteristics with the audience's appreciation value, thus constantly cultivating students' individuality. Through the long-term practice and learning, the author has uploaded songs with multiple innovative performance styles to the learning column and students may download them for independent learning. At the same time, multiple means of teaching also create a strong learning atmosphere, stimulate students' learning interest and bring them a strong sense of engagement.

The column of tutoring and exchange allows in-depth exchange and learning for teachers and students concerning the songs and performance skills of Guzheng. Students may give an online performance and make corrections after receipt of timely feedback and suggestions from teachers. In addition to the assessment of learning effect, there may be online exchanges among students and between students and teachers. There are many means of online exchange, including video presentation, voice communication, text or pictures, etc. The range and content of exchange may be extensive. For instance, teachers may play a Guzheng song and ask students to delivery their opinions after listening. The network teaching changes tentativeness of students and allows them to give personal opinions freely. Then, the teachers' targeted interpretation will further incite valuable opinions from students.

The column of video and audio production appreciation includes many domestic and foreign Guzheng masterpieces. Students may download them for self-learning. Students may search songs 
online by the author, performance time, performance place or school, or download them to an intelligent mobile equipment.

The online teaching system of Guzheng also includes the certification of learners' identity, upgraded learning information and resources of Guzheng, software upgrading and sub-management system, etc., providing an all-round teaching mode for internet online teaching of Guzheng. The internet online teaching system of Guzheng has attracted attentions from Guzheng lovers ever since it was launched. In this system, students will acquire not only much knowledge about learning of Guzheng, but also all kinds of performance skills. Furthermore, they can also appreciate the masters' representative onsite performances. After learning with this system, students' performance skills and exchanges are all greatly improved, in addition to the techniques in playing Guzheng.

The internet online teaching system of Guzheng provide students with a good learning environment and atmosphere. Learners may utilize this system to learn knowledge and skills. In case of a membership, he/she may have a one-to-one exchange with teachers and submit their problems. The internet online learning of Guzheng provides a satisfactory learning environment as well as a solid foundation for extensive network teaching.

\section{Conclusion}

To sum up, the remote online teaching system of Guzheng offers a new teaching concept. The innovative point of modern online teaching of Guzheng lies in its online spreading of Guzheng education, so that netizens may get an understanding of Guzheng, disseminate the culture and art of Guzheng and help introduce Guzheng to the world. Therefore, it is of great significance to development of current online Guzheng education.

\section{References}

[1] Lang Juanjuan. Application of Flipped Classroom in Higher Normal Ancient Zither Teaching[J]. Hundred Schools in Arts,2016, (z1):332-334.

[2] Song Yanbin. Discussion on Construction and Teaching Reform of Music Online Open Course in Schools [J]. Journal of Huaihua University,2018, 37(6):125-128.

[3] Cheng Jing, Peng Qian, Liu Tong, Chen Xiaoyan. Online Teaching in Local Colleges and Universities in the Era of Mobile Internet: Challenge, Breakthrough and Reconstruction [J]. Journal of Hubei Engineering University, 2020, 40(3):07-77.

[4] Zhang Yiyue. Chance and Challenge: Cloud Class of the Major of Higher Education of Music in the AntiEpidemic Period [J].China Music Education,2020(5):31-35. 